sciendo

\title{
Supervaluationism and Necessarily Borderline Sentences
}

Pablo Cobreros

University of Navarra

Disputatio Vol. 3, No. 25

November 2008

DOI: $10.2478 /$ disp-2008-0009

ISSN: 0873-626X 


\title{
Supervaluationism and necessarily borderline sentences
}

\begin{abstract}
The supervaluationist theory of vagueness is committed to a particular notion of logical consequence known as global validity. According to a recent objection, this notion of consequence is more problematic than is usually thought since i) it bears a commitment to some sort of bizarre inferences, ii) this commitment threatens the internal coherence of the theory and iii) we might find counterexamples to classically valid patterns of inference even in the absence of a definitely-operator (or similar device). As a consequence, the supervaluationist theory itself is in trouble. This paper discusses the objection.
\end{abstract}

Pablo Cobreros

University of Navarra

\section{Keywords}

Logical consequence, vagueness, supervaluationism.

\section{Introduction}

Semantic unsettledness looks like a prima facie appealing explanation of what vagueness consists in. The idea is that the facts that determine the meaning of a vague expression do not settle for a range of cases whether the expression applies. Where Tim is a borderline case of the predicate 'thin', the facts that determine the meaning of the predicate do not settle the question of whether Tim is thin. The supervaluationist theory of vagueness provides a picture to understand this sort of semantic unsettledness. ${ }^{1}$ The basic idea is that a vague expression can be made precise in different ways all them consistent with the use we make of the expression. The sentence 'Tim is thin' will be true in some ways of making 'thin' precise and false in some other ways. Since all these ways of making 'thin' precise are consistent with the use we make of the expression, our use does not decide between them.

\footnotetext{
1 The classical paper on the supervaluationist theory is Fine's 1975. Keefe $2000 \mathrm{~b}$ provides a detailed exposition and defence of the theory.

Disputatio, Vol. III, No. 25, November 2008
} 
Supervaluationism is a truth-gap theory. This fact is conveyed in the slogan that 'truth is supertruth': a sentence $A$ is (super-)true just in case it is true in every way of making $A$ 's vague expressions precise. Since some sentences are true in some ways of making precise the relevant expressions and false in some other ways, neither the sentence nor its negation are (super-)true. With the reasonable assumption that falsity is the truth of the negation, these sentences are neither true nor false.

A precisification is a way of making precise all the vague expressions of a given language. Formally speaking, a precisification is analogous to a classical interpretation. An admissible precisification is a precisification respecting certain constraints that might hold between sentences. For example, where Tom is a bit thinner than Tim, any precisification that counts Tim as thin should also count Tom as thin. As a consequence, the conditional 'if Tim is thin then Tom is thin' is supertrue regardless of the truth-value of either component-sentence, since the consequent will be true in any precisification in which the antecedent is true. The accommodation of this sort of penumbral connections is often invoked as a reason in favour of supervaluationist semantics over a truth-functional approach.

Despite its non-classicality, supervaluationist semantics provides a notion of logical consequence that is akin to classical logic. Since validity is a matter of necessary preservation of truth and for the supervaluationist truth is supertruth, for the supervaluationist logical consequence is a matter of necessary preservation of supertruth. This notion of consequence is known as global validity: $A$ is a global consequence of a set of sentences $\Gamma$ just in case, for every interpretation, if all the members of $\Gamma$ are true in all admissible precisifications, then $A$ is true in all admissible precisifications. Global validity coincides with classical consequence in the absence of non-classical operators. ${ }^{2}$

Supervaluationist semantics allows for a natural extension of the classical language: we might introduce a definitely operator (' $\mathrm{D}$ ' henceforth) with the following definition: a sentence $\mathrm{D} A$ is true just in case $A$ is true in every precisification. ${ }^{3}$ The $\mathrm{D}$-operator is a natural

\footnotetext{
${ }^{2}$ See Fine (1975: 283-4) and Keefe (2000b: 175-6). The result is restricted to single-conclusions consequence relations.

${ }^{3}$ The accommodation of higher-order vagueness requires a refinement of this definition. The standard procedure is to introduce an admissibility-relation between
} 
extension of the classical language in the present context since it allows us to talk about borderline cases: A is a borderline sentence just in case $\neg \mathrm{D} A \& \neg \mathrm{D} \neg A$. The operator is an object-language expression of supertruth. However, once the D-operator is in play, global validity no longer coincides with classical logic. In particular, we might find counterexamples to classically valid rules of inference such as reductio ad absurdum, conditional proof, contraposition and argument by cases. ${ }^{4}$

Here ends the brief introduction on supervaluationism and logical consequence. The following section considers an objection to the supervaluationist theory based on the commitment of this theory to global validity and on the existence of necessarily borderline sentences.

\section{An objection concerning global validity}

In his 2008 paper, Martin Montminy raises an objection against the supervaluationist theory. The objection is based on the supervaluationist commitment to global validity and is articulated through three related claims: first, that global validity bears a commitment to a particular sort of counterintuitive inferences, second, that this commitment threatens the internal coherence of the theory and third, that supervaluationism is committed to counterexamples to classically valid patterns even in the absence of a definitely-operator or similar device. This section presents and discusses each of these claims.

\subsection{Counterintuitive inferences}

Objection: The objection begins with the observation that some sentences seem to express a borderline case independently of how the world is. Some examples include 'Darts is a sport', 'Skis are a vehicle' and 'A 34-year-old woman is in her early thirties' (Montminy 2008: 63). If a sentence of this sort expresses a borderline case, it expresses a borderline case in every possible world. Call these sentences necessarily borderline.

A borderline sentence is, according to supervaluationism, neither true nor false. Thus, a necessarily borderline sentence is necessarily

precisifications (see Williamson, 1994:157). Since the present discussion is independent from that of higher-order vagueness, I omit the point for clarity.

${ }^{4}$ See Williamson (1994: 151-2). 
neither true nor false. Since validity is a matter of necessary preservation of truth, supervaluationists seem compelled to conclude that anything is a valid consequence of a necessarily borderline premiss (since it is impossible for the premiss to be true). So, in particular, Montminy claims that supervaluationists are committed to the validity of the following argument,

(A)

A 34-year-old woman is in her early thirties

$\therefore$ A 33.9-year-old woman is not in her early thirties.

Response: Both premisses of the objection, that there are necessarily borderline sentences and that validity is a matter of necessary preservation of truth, look quite reasonable claims. What I find at least questionable is the reading of necessity involved in the definition of validity.

W-validity: An argument $C$ is $\mathrm{W}$-valid iff in every possible world $w$, if all the premisses are true in $w$ then the conclusion is true in $w$.

I-validity: An argument $C$ is I-valid iff in every interpretation $i$, if all the premisses are true in $i$ then the conclusion is true in $i$.

Validity is a matter of necessary preservation of truth, but the reading of 'necessary' in the argument above is not the usual one in discussions about supervaluationism and validity. That an argument necessarily preserves truth is usually read as that it preserves truth, not in every possible world, but in every interpretation; that is, validity is usually read as I-validity. ${ }^{5}$ However, when validity is read in this way, argument (A) above is no more valid than the following argument-schema:

(B)

$$
\therefore \stackrel{p}{\neg q}
$$

A remark: Still, there is a correct insight in the objection above. Since validity is a matter of necessary preservation of truth, an argument with a borderline premiss is truth-preserving. If the meaning of that premiss is kept fixed through all interpretations, the argument must be necessarily truth-preserving. This insight might be expressed

\footnotetext{
${ }^{5}$ See, for example, Fine 1975, Williamson (1994: ch. 5), Keefe 2000a and 2000b, and Varzi 2007.
} 
if we have the resources to speak about borderline cases; and the standard way to achieve this expressiveness is by introducing a Doperator. The set of sentences $\{\neg \mathrm{D} A \& \neg \mathrm{D} \neg A, A\}$ is not satisfiable for the supervaluationist, since there is no interpretation in which these two sentences are true in every precisification. Thus, the following argument is globally valid (reading validity as I-validity),

(C)

$$
\therefore \quad \stackrel{\perp}{ } \quad \begin{aligned}
\mathrm{D} A \& \neg \mathrm{D} \neg A, A\} \\
\end{aligned}
$$

This shows that argument (A) would be I-valid if we understand that the meaning of the premiss is kept fixed such that $\mathrm{A}$ is a borderline sentence in every interpretation. But if an expression keeps its meaning fixed across different interpretations, then it no longer belongs to the extra-logical part of the vocabulary. The new logical constant might very well increase the expressiveness of the language. This remark will be of interest in section 2.3.

Conclusion: The conclusion of this first section is that the argument for the supervaluationist commitment to the validity of arguments like (A) works just under a particular notion of validity that is not the standard one, at least in discussions about validity in the supervaluationist theory.

\subsection{Internal coherence}

Objection: In the second place Montminy argues there are internal reasons for the supervaluationist to regard the inference (A) as invalid. The putative reason is that the conditional,

(*) If A 34-year-old woman is in her early thirties, then a 33.9-year-old woman is not in her early thirties,

expresses a penumbral falsehood for the supervaluationist. This putative commitment to the falsehood of $(*)$ 'should force them [supervaluationists] to regard arguments such as (A) as invalid. Hence, not only does global validity have counterintuitive consequences, but it also jeopardizes the internal coherence of supervaluationism' (Montminy 2008: 64).

Response: The problem with this argument is that its main premiss is false. To make things more perspicuous, consider an ordered 
series of sentences from 'A 40-year-old woman is in her early thirties' to 'A 30-year-old woman is in her early thirties' each one differing from its successor in the obvious way. The first sentence is clearly false, the last is clearly true. Tag each sentence as $F(n . m)$, depending on its position in the series; for example, 'A 33.9-year-old woman is in her early thirties' is tagged as $F(33.9)$. Now supervaluationists are committed to the truth of conditionals like ' $F(34) \rightarrow F(33.9)$ ' since in every precisification in which ' $F(34)$ ' is counted as true, ' $F(33.9)$ ' should also be counted as true. In an analogous way, supervaluationists are committed to the falsehood of sentences like ' $\neg(F(34) \rightarrow$ $F(33.9))$ '. But for supervaluationists there is no penumbral connection justifying the falsehood of,

(*) F(34) $\rightarrow \neg \mathrm{F}(33.9)$

since by the own assumption that ' $F(34)$ ' is a borderline sentence it follows that there are admissible precisifications in which $(*)$ is true (take just one in which ' $F(34)$ ' is false). If supervaluationism were committed to the falsehood of this sort of sentences, then the view would be straightforwardly incoherent.

Conclusion: Though I still think that arguments such as (A) should not be regarded as valid by supervaluationists, the conclusion of this second section is that Montminy's argument fails to show that the validity of (A) jeopardizes the internal coherence of supervaluationism.

\subsection{Classical rules of inference}

Objection: In the third place, Montminy argues that some classically valid rules of inference fail for the supervaluationist notion of validity even in the absence of a 'definitely'-operator. Counterexamples to classically valid rules of inference this time involve necessarily borderline sentences.

[Assume] that ' $A$ ' is a necessarily borderline sentence (say, 'Darts is a sport') and ' $C$ ' a contingently true sentence (say, 'Darts is popular in pubs in Britain'):

(1) ' $A$ ' globally entails ' $C$ ', but 'not- $C$ ' does not globally entail 'not- $A$ ', for it is possible for 'not- $C$ ', but not 'not- $A$ ', to be true. Hence, contraposition is violated (Montminy 2008: 65). 
Other putative counterexamples against conditional proof, argument by cases and reductio follow in a similar way.

Montminy notes that these failures are analogous to the failures in the presence of a 'definitely'-operator and considers a response of Keefe's to this particular case. Keefe points out that all the counterexamples make use of the inference from $A$ to $\mathrm{D} A$ and suggests a new set of rules for those arguments involving this inference. ${ }^{6}$ But Montminy argues that Keefe's defence cannot be maintained, 'for the violations of classical inferential rules described above involve arguments containing no non-classical expressions: even when the D operator is absent, the classical rules fail to apply.' (Montminy 2008: 66).

Remark: The claim that there might be counterexamples to classical rules in the absence of the D-operator (or similar device) is striking since exactly the opposite has been proved. ${ }^{7}$ This divergence shows that there are two different notions of validity in play, $\mathrm{W}$ validity and I-validity. The second reading is the one assumed in Fine's and Keefe's proof that classical and supervaluationist consequence coincide in absence of ' $\mathrm{D}$ '.

Response: If we read validity as I-validity, there are two ways in which we might interpret the role of ' $A$ ' in counterexample (1):

a) ' $A$ ' is read as a schematic letter standing for any sentence whatsoever.

b) ' $A$ ' keeps its meaning constant expressing a borderline case in every interpretation.

In the first case, the counterexample does not work since ' $A$ ' ranges over any interpretation and, thus, it is not the case that $C$ follows globally from $A$.

In the second case, ' $A$ ' is treated as a new logical constant. This addition to the vocabulary might increase the expressiveness of the language and in this particular case it does. It is no wonder that the

\footnotetext{
${ }^{6}$ Keefe (2000: 178-81). Though I think that Keefe's particular strategy does not work (since the proposed restriction would render an incomplete deductive system for global validity) it seems to me that Keefe's general approach is basically right. We might produce a sound and complete system of deduction for global validity by adding a new rule (From $\Gamma \vdash A$ infer $\Gamma \vdash \mathrm{D} A$ ) and placing suitable restrictions on the use of other rules; this fact is shown and discussed in an author's unpublished manuscript.

${ }^{7}$ See Fine (1975: 283-4) and Keefe (2000: 175-6). The proof works just for single-conclusions consequence.
} 
counterexamples pointed out by Montminy mirror those involving ' $D$ ', since ' $D$ ' increases the expressiveness allowing us to talk about borderline sentences, and ' $\mathrm{A}$ ' is treated in the putative counterexample with the fixed meaning of a borderline sentence. Under this light, the claim that classical rules fail to apply in the absence of nonclassical expressions becomes suspicious: 'A' itself is treated as a nonclassical expression.

Conclusion: The conclusion of this third section is that Montminy's argument for the failure of classical rules in the absence of ' $D$ ' rests again on the particular reading of validity as $\mathrm{W}$-validity.

\section{I-validity vs. W-validity}

Validity is a matter of necessary preservation of truth. The objections of Montminy's are based on the particular reading of this rough definition as $\mathrm{W}$-validity. However, I-validity is the standard reading; not just in discussions concerning vagueness, but when talking about logical consequence in general. In fact, since Tarski's influential work in the thirties, I-validity has become the standard usage in Logic textbooks. In addition to this important reason, I point out now very briefly why it seems to me that I-validity is to be preferred.

From the early beginnings of logic, logical consequence has been thought as something tied to the form of an argument. A characteristic step in Aristotle's syllogistic is the generalization from arguments formulated in a natural language to the form of these arguments. Though some particular forms of argument identified by Aristotle might look controversial nowadays, the crucial thing is that this sort of generalization proceeds by the identification of expressions whose meaning remain constant and expressions acting as a sort of placeholders. This view relating logical consequence to the form of an argument has remained in its essence in contemporary views as in the influential work of Bolzano and Tarski, where validity is the absence of counterexamples in any of the admissible ways in which we might interpret the non-logical vocabulary.

The problem with $\mathrm{W}$-validity is that, according to this reading, logical consequence has little to do with the form of an argument. Take a pair of simple sentences $A$ and $B$, the first expresses a metaphysical falsehood and the second a contingent truth. The arguments: $A$ therefore $\perp$ and $B$ therefore $\perp$, are structurally identical; however, 
the first is valid and the second is not. In a similar way, take two simple sentences $A$ and $B$ expressing metaphysically incompatible propositions (say, 'Tim is a man' and 'Tim is a dog'). The argument $\{A, B\}$ therefore $\perp$ should be valid even if there is nothing left to analyse about the structure of $A$ and $B$.

The connection of validity and form is an important feature of logic. One of the primary tasks of logic is to provide systematic ways to decide whether a given argument is valid. A proof theory for a notion of logical consequence consists on a set of rules that can be applied just looking at the form of sentences. But when validity is dissociated from form in the way done by $\mathrm{W}$-validity, the task of finding suitable proof theory looks like a hopeless search.

The supervaluationist might look at the situation this way: the balance is already heavily tilted towards I-validity. For the objection to have any force, a better defence of $\mathrm{W}$-validity is required. In the meantime she should not be worried about the existence of necessarily borderline sentences. ${ }^{8}$

Pablo Cobreros pcobreros@unav.es Dept. of Philosophy University of Navarra 31080 Pamplona, Spain

\section{References}

Fine, Kit. 1975. Vagueness, Truth and Logic. Synthese 30: 265-300.

Keefe, Rosanna. 2000a. Supervaluationism and Validity. Philosophical Topics 28: 93-105.

Keefe, Rosanna. 2000b. Theories of Vagueness. Cambridge: Cambridge University Press.

Montminy, Martin. 2008. Supervaluationism, Validity and Necessarily Borderline Sentences. Analysis 68: 61-67.

Varzi, Achille. 2007. Supervaluationism and its Logic. Mind 116: 633-676. Williamson, Timothy. 1994. Vagueness. London: Routledge.

${ }^{8}$ This paper was made possible by a postdoctoral scholarship from the Government of the Basque Country (BFI07.235). The research was also supported by a project of the Government of Spain (ref. HUM2005-05910/FISO). The paper was written during my stay in The Institute of Philosophy, London. I am particularly grateful to Will Bynoe, Catherine Wearing, and Barry Smith. I am also grateful to Nick Jones, María Cerezo and Paloma Pérez-Ilzarbe for several comments and discussion. 